\title{
CARACTERÍSTICAS DE LA REFLEXIÓN DE ESTUDIANTES DE DERECHO DE LA UNIVERSIDAD SANTO TOMÁS DE BUCARAMANGA, EN LAS INTERACCIONES GON SUS ASESORES Y MONITORES DURANTE LAS PRÁGTICAS DE CONSUITORIO JURÍDICO
}

\author{
Miguel López Gómez ${ }^{1}$ \\ Universidad Santo Tomás - Bucaramanga
}

\begin{abstract}
Resumen
El propósito de esta investigación fue identificar las características de la Reflexión en las Prácticas de Consultorio Jurídico, conocer el nivel de profundidad de esta reflexión, los conocimientos implicados en la acción de Asesoría Jurídica y la relación con los conocimientos del plan de estudios de Derecho y con nuevos conocimientos. Busca conocer cómo está relacionada la reflexión con actitudes como la motivación, valores de respeto y atención a los usuarios del Consultorio. Las categorías que se trabajaron fueron reflexión, interaccionismo simbólico y práctica profesional. El abordaje metodológico se realizó desde una perspectiva de las investigaciones en ciencias sociales cualitativa, hermenéutica con observación no participante. El análisis de datos es determinado por el interaccionismo simbólico y la pedagogía de la comprensión. Se analizaron tres casos de derecho: civil, penal y laboral. La investigación permitió concluir que la práctica del consultorio jurídico es un verdadero prácticum reflexivo. La relación docente - practicante presenta un nivel de compañerismo. Los monitores influyen en los niveles de reflexión. Son vivenciados los valores éticos y el buen desempeño teórico de los estudiantes. Se hace una propuesta metodológica para la Facultad de Derecho y el Departamento de Humanidades el diseño de prácticum reflexivos como estrategia didáctica.
\end{abstract}

\section{Palahras clave}

Prácticas profesionales, hermenéutica, interaccionismo simbólico, prácticum reflexivo, consultorio jurídico, dimensión teleológica, cognitiva, metódica, formal.

\section{CHARACTERISTICS OF THE REFLECTIONS OF LAW STUDENTS OF THE UNIVERSITY SANTO TOMÁS FROM BUGARAMANGA, ABOUT THE INTERACTIONS WITH THEIR ADVISERS AND MONITORS DURING THE PRAGTICES OF LEGAL CONSULTING}

\section{Alstract}

The intention of this research was to identify the characteristics of the Reflections about the Practices of Legal Consulting, to know the level of depth of these reflections, the knowledge implied in the action of Legal Consultancy and the connection with the knowledge of the Law curriculum and with new knowledge. It aims to find out how the reflections are related to attitudes like motivation, values of respect and attention to the users of the Law Consulting services. The categories worked on were reflections, symbolic interactionism and professional practice. The methodological approach was carried out from a social sciences perspective that was qualitative, hermeneutical and with nonparticipant observation. Data analysis is determined by the symbolic interactionism and the pedagogy of understanding. Three cases were analyzed: civil, criminal and labor law. The research took us to the conclusion that the practice of legal consulting is a true reflective practicum. The relationship teacher - student in practice presents a comradeship level. The instructors influence in the levels of reflections. The students experience ethical values and good theoretical performance. A methodological proposal is carried out for the Faculty of Law and the Department of Humanities about the reflective design of practicum as an educational strategy.

\section{Key Words}

Professional practices, hermeneutics, symbolic interactionism, reflective practicum, legal consulting, teleological, mental, methodical and formal dimension.

\footnotetext{
1 Docente de tiempo completo de la Universidad Santo Tomás de Bucaramanga. Candidato a Doctor en Educación, Currículo, Profesorado e Instituciones Educativas de la Universidad de Granada - España. Magíster en Evaluación en Educación y Especializado en Docencia Universitaria de la Universidad Santo Tomás Bucaramanga. Licenciado en Filosofía y Ciencias Religiosas, Universidad Santo Tomás Bogotá. milogo01@ hotmail.com
} 


\section{Introducción}

El trabajo de investigación se realiza con el Dr. Ernesto Sánchez Jerez, como investigador principal.

La articulación entre la teoría y la práctica, para la formación de profesionales reflexivos es uno de los desafíos de la Educación Superior en el siglo XXI. Los sistemas educativos fundamentados en los modelos científicos de la modernidad, centrados en la racionalidad técnica e instrumental, han separado la teoría de la práctica, y dejado a un lado la epistemología de la acción, en los procesos de formación de los estudiantes en sus prácticas en relación con la resolución de los problemas inherentes al ejercicio de sus disciplinas, en las interacciones con sus Docentes, asesores y monitores en las prácticas profesionales.

La modernidad dio origen a una racionalidad económica, técnica, industrial, positivista que separó lo científico de la práctica y se polarizó en lo general y en lo universal para originar la ineficiencia, la deshumanización y la separación de la reflexión en las prácticas profesionales.

Esta investigación se realiza para buscar identificar las características de la reflexión en las prácticas de consultorio Jurídico, conocer el nivel de profundidad de esta reflexión, los conocimientos implicados en la acción de asesoría Jurídica y la relación con los conocimientos del plan de estudio de Derecho y con nuevos conocimientos. Busca conocer cómo está relacionada la reflexión con actitudes como la motivación, valores de respeto y atención a los usuarios del Consultorio.

La Universidad Santo Tomás pretende conocer sus propios procesos de formación integral, fundamentalmente desde aquellas metodologías que en la actualidad se consideran como las más adecuadas para la formación de
Profesionales Integrales. Es el caso de la formación a través de la reflexión sobre la práctica Profesional, impulsada en 1982, por el Dr. Donald Schön desde la División para el estudio y la Investigación en Educación en Massachusetts Institute of Technology.

En la Universidad Santo Tomás, varias facultades forman a sus estudiantes en la reflexión desde la práctica profesional. La primera en implementarla fue la Facultad de Derecho fundada en 1973. Por eso fue escogida para el trabajo de Investigación.

La reflexión sobre la práctica es un momento crítico en la formación del profesional, ya que allí como en una creación, se configuran las grandes competencias incubándose los hábitos y las semillas más fructíferas de conocimientos, actitudes y habilidades del futuro profesional integral. El conocer las características de esta reflexión orienta a la Universidad, sobre la manera como se contribuye a la obtención de la misión de la USTA, empeñada en que sus egresados "respondan de manera ética, creativa y crítica a las exigencias de la vida humana y estén en condiciones de aportar soluciones a la problemática y a las necesidades de la sociedad y del país". (Misión. USTA)

Las categorías que se trabajaron en esta investigación son las de reflexión, interaccionismo simbólico y práctica profesional. La reflexión un elemento definitorio de la especie humana, puede caracterizarse en muchos niveles y algunos de ellos pueden no estar presentes en algunas acciones verdaderamente humanas. Según John Dewey, la relación entre acciones y consecuencias a veces no se da por la inercia, la rutina o capricho y menos la reflexión que busca indagar sobre lo que une la acción y sus consecuencias.

En la investigación en prácticas profesionales reflexivas se analizaron tres casos, 
uno de derecho civil, otro de penal y un tercero de laboral.

El análisis del tipo de reflexión se hizo mediante la aplicación de un marco categorial que permite clasificar el nivel de reflexión que se da en las interacciones de los practicantes en las interacciones con los docentes, los monitores, los asesores y los usuarios en el Consultorio Jurídico.

Como resultado, se presentan las conclusiones y las propuestas pedagógicas como el diseño de prácticum reflexivos, estrategia didáctica y aportes a la formación de Profesionales reflexivos en la Facultad de Derecho.

\section{Referentes Teóricos}

\section{Antecedentes de la investiga- ción}

Como antecedentes investigativos en prácticas reflexivas en el Consultorio Jurídico, no se han realizado trabajos en este campo en la Universidad Santo Tomás.

A nivel local se pueden citar algunas tesis de grado sobre implementación y mejora del Consultorio Jurídico de la Universidad Industrial de Santander. "Sistema de Intranet para el apoyo al trabajo colaborativo entre los miembros de la comunidad del Consultorio Jurídico de la Universidad Industrial de Santander. Intranet. C.J. 2005". Realizado por Lizett Gereda Pico y Víctor Hugo Cárdenas, Director: Luis Ignacio González Ramírez. Esta investigación nos proporcionó una nueva perspectiva para el trabajo didáctico en el Consultorio.

Encontramos dos investigaciones en la Escuela de Derecho y Ciencias Políticas en la U.I.S. Una sobre: "Creación y puesta en marcha del centro de conciliación adscrito al Consultorio Jurídico de la Escuela de Derecho y Ciencias Políticas de la Universidad Industrial de Santander." (2005). Realizado por César
Augusto Quijano Quiroga y Jenny Rocío Suárez Quintero, dirigido por la Dra. María Victoria Gómez Posse. Y la otra sobre Consultorio Socio-Jurídico como estrategia pedagógica, realizado por Rubén Hernando Morales, dirigida por Leonardo Moncayo Rosales, CEDEUIS 2000, por privilegiar espacios dialogales, por apostarle al mundo de la vida y sobre todo por sus aportes a la dimensión teleológica que prioriza los fines sociales y humanos del Derecho.

A nivel Nacional encontramos un trabajo de Investigación sobre: "Interacciones profesor- estudiante en procesos formativos de la facultad de Odontología de la Pontificia Universidad Javeriana (2004) realizado por Dra. Francina María Escobar Arregocés, y los Procesos Pedagógicos y sus vicisitudes, reflexiones y aproximaciones de la Universidad EAFIT de Medellín (2005), realizado por Jeannette Lerner Matiz. Artículo científico. Revista Universidad EAFIT, Volumen 41, No. 139 (2005).

El trabajo Investigativo sobre la relación Profesor- Estudiante de la Universidad Javeriana nos aportó ideas en lo referente a la relación Profesor- Estudiante-. EI trabajo sobre los procesos pedagógicos de EAFIT, nos proporcionó orientaciones acerca de considerar la necesidad de considerar los diálogos interdisciplinarios y la trasmisión de valores e imaginarios.

A nivel Internacional, se encontró una investigación sobre: "Formación Docente y práctica en el aula" adelantada entre 1996 y 1997, en el Departamento de Ciencias de la Educación, de la Universidad de Río Cuarto en la República Argentina, realizado por Teresita de Rivas. Fue un trabajo investigativo muy estimulante pues en él se trató de resignificar la reflexión sobre la acción como tarea permanente y necesaria para la formación de los futuros educadores. Esta re-significación puede extenderse a otras disciplinas profesionales. 
El interaccionismo simbólico y la reflexión en la práctica profesional

Se plantea desde el interaccionismo simbólico de Herbert Mead y del pensamiento de $\mathrm{H}$. Blumer establecer la naturaleza social del conocimiento y la importancia de la construcción del saber profesional a partir de sus experiencias en las prácticas profesionales y de las interacciones con los docentes, monitores y asesores buscar responder de manera eficiente a los problemas del ejercicio profesional. Se considera al profesional como un continuo aprendiz en razón a su condición de ser con los otros y en una interacción dual en los procesos pedagógicos.

El interaccionismo simbólico ubica al hombre como un ser necesitado de los demás por su indeterminación genética. Para poder desarrollarse necesita de una cultura que le permita entender y hacerse entender en la vida social y que lo habilite para transformar el mundo físico en el que está. Esta cultura la obtiene en la interacción con los demás a través de los símbolos.

\section{Pedagogía de la reflexión desde la acción}

La práctica profesional es la competencia de una comunidad de prácticos que comparten las tradiciones de una profesión según Schön. Si bien esta práctica se actualiza con la obtención del título Profesional, se da también en el proceso de formación Universitaria, con la ayuda de expertos. En este caso el estudiante realiza prácticas acompañado de un profesional experimentado ya sea directamente o después, en la reflexión que se hace por lo realizado. Este proceso se denomina "prácticum reflexivo", cuya principal característica es la posibilidad de creación ilimitada sin consecuencias inmediatas para el usuario. A este nivel pertenecen las interacciones del estu- diante con su monitor y su asesor en el Consultorio Jurídico.

En la investigación en prácticas profesionales reflexivas se analizaron tres casos, uno de derecho civil, otro de penal y un tercero de laboral.

El análisis del tipo de reflexivo se hizo mediante la aplicación de un marco categorial que permitió clasificar el nivel de reflexión que se da en las interacciones de los practicantes e las interacciones con los docentes, monitores, asesores y usuarios en el Consultorio Jurídico.

Como resultados, se presentan las conclusiones y las propuestas pedagógicas como el diseño de prácticum reflexivos, estrategia didáctica y aportes a la formación de Profesionales reflexivos en la Facultad de Derecho.

\section{Metodología}

La investigación pertenece a las Ciencias Sociales, histórico-hermenéuticas, y crítico-sociales, su finalidad es interpretar el mundo social para comprenderlo y realizar una ubicación más adecuada, y no para manipularlo como en las ciencias empírico-analíticas, no para buscar la emancipación, como en las ciencias crítico-sociales (Alvarado y otros, 1992). El principio fundamental, del interaccionismo simbólico que orientó la metodología utilizada es el de la "concepción que hacen los actores sobre el mundo social, constituye en el fondo el objeto social de la investigación en la ciencias sociales" (Alvarado y otros, 67). Y esta concepción se forma por medio de la reflexión de cada sujeto agente, reflexión que se transforma en las interacciones con los demás y en aquellas que son específicamente educativas.

La reflexión es una variable que no se presenta sola y aislada, al modo de los elementos físicos y, por lo tanto, representable por un signo matemático, sino que es el resultado de un sinnúmero de 
relaciones entre personas, entre ellas y los objetos, con el contexto. "La naturaleza íntima, estructural-sistémica, de los procesos más típicamente humanos no es captada por las técnicas matemáticas señaladas" (Martínez M., s.f., p.31). Tampoco es objeto de análisis al modo de las ciencias empírico analíticas ya que éstas trabajan con elementos que carecen de relación entre sí y que se comportan linealmente, (Martínez M., apoyándose en Bertalanffy, p.34), y la reflexión es el resultado de la interacción de personas que realizan "acciones humanas" con toda la riqueza y complejidad que ello implica. Es la concreción de los significados dados por las interacciones humanas, significados que no solamente son objetivos sino también subjetivos; y éstos nacen de su relación con la totalidad de la interacción, de sus motivos porque y sus motivos para.

Al contrario de la investigación cuantitativa, no se trabajó con experimentos, que contradicen la dignidad de la persona al reducirla a objeto, sino con observaciones. En este caso, con observaciones no participantes, que trataron de no influir en nada en el transcurso de las interacciones de los practicantes con sus asesores y monitores principalmente.

El propósito de la investigación fue encontrar la realidad de lo que es la reflexión de algunos practicantes concretos de la Universidad Santo Tomás, en su carácter singular y complejo, llegándose a las proximidades de una verdad de lo particular que pueda ser transferida a casos parecidos.

“Los investigadores de orientación interpretativa se centran en la descripción y comprensión de lo que es único y particular del sujeto más que en lo generalizable; pretenden desarrollar conocimiento idiográfico y aceptan que la realidad es dinámica, múltiple y holística..." (Arnal y otros, 1992, p. 41).
En la orientación de la investigación seguimos un sabio consejo de Herbert Blumer:

“Un pequeño número de individuos, tomados como un grupo recurso y de discusión, es más valioso, muchas veces, que cualquier muestra representativa" (Blumer, 41).

Este grupo lo constituyeron la Directora del Consultorio Jurídico y dos Monitores. Ellos nos ayudaron a seleccionar los casos para nuestro estudio, se tuvo como criterios la pertinencia y un grado mediano de dificultad, según la recomendación de Goetz y Lecompte: "Aquí, el investigador desarrolla un perfil de los atributos que posee el caso intermedio para, a continuación, encontrar un ejemplar" (p. 101). El grupo, con los investigadores, escogió seis casos: dos de Derecho Civil, uno de Público Administrativo, uno Penal, uno Laboral y una Conciliación. A todos les filmamos asesorías y entrevistas, pero al final sólo tuvimos en cuenta uno de Civil, otro de Penal y un tercero de Laboral, porque cumplieron plenamente los requisitos de asesorías que deseábamos investigar. La unidad de análisis de la investigación la constituyó cada uno de los tres casos en su proceso de ayuda jurídica. Está constituida por las interacciones del practicante con el usuario, con el Monitor y con el Asesor, además de las entrevistas con los investigadores.

Los datos objeto de análisis son determinados por el interaccionismo simbólico: “Un marco teórico amplio, como el interaccionismo simbólico, precisa, simplemente, que los datos reflejen intercambios entre individuos" (Goetz y Lecompte, p. 81). Como aquí se trata de la reflexión sobre la acción jurídica en el Consultorio, lo que comúnmente refleja intercambio entre los individuos es el diálogo oral entre el estudiante y el Usuario, su Asesor y su Monitor. Sin embargo, esto no se dio en todos los casos. Fue válido para el caso de Penal, pero en los de Laboral y Civil, los 
intercambios se realizaron fundamentalmente a través de escritos y los diálogos se redujeron a referencias a lo escrito. En cada caso se analizaron los elementos que contribuyeron a la identificación de las características de la reflexión.

Se recurrió, entonces, a la lectura y a la observación para tratar de comprender la experiencia humana, objeto genérico de la investigación histórica hermenéutica (Alvarado, Módulo 1, 76). Respecto a la observación, ésta fue de naturaleza no participante, con el objeto de no interferir en el proceso de reflexión del estudiante con su asesor o su monitor.

"Al investigador le interesa cómo tiene lugar la interacción cuando no está presente un observador externo. En consecuencia, los observadores no participantes intentan implicarse lo menos posible en los hechos que están registrando. Ello les obliga a situarse, y a colocar sus equipos de observación, en el lugar menos intrusivo posible" (Goetz y Lecompte, p. 155).

Se realizó de manera que en el diálogo estudiante-asesor, estudiante-monitor, sólo estuviera presente una cámara de video que grabó voz e imagen de la in- teracción reflexiva. Lo obtenido con la cámara se pasó a texto escrito, para su posterior análisis. Como habíamos dicho, la observación tuvo relevancia más que todo en el caso de Penal. En los de Civil y Laboral, toda la importancia la asumió el texto escrito por medio del cual el practicante presenta su trabajo y en el cual el Monitor, principalmente, escribe sus notas con comentarios y correcciones. Estos textos escritos fueron concienzudamente analizados por los investigadores. Además, los tres practicantes fueron entrevistados por los investigadores para afinar aspectos de la reflexión realizada en su práctica profesional.

Para analizar los textos escritos, las entrevistas y las observaciones de las asesorías con el fin de caracterizar la reflexión, se construyeron categorías para la supercategoría "Reflexión". Este paso constituyó una gran dificultad para los investigadores, pues los autores que trabajan la reflexión como Dewey, Schön y Brockbank y McGill no presentan suficientes categorías reflexivas: Dewey se limita a la reflexión rutinaria o consciente y a la irresponsable o con consecuencias, y los demás se refieren a caracterizaciones temporales con relación a la acción.

\section{CATEGORIAS DE ANARUSIS}

\begin{tabular}{|c|c|c|c|c|c|c|}
\hline DIMEN. & REFLEXIÓN & Niv.1 & Niv.2 & Niv.3 & Niv.4 & $\begin{array}{c}\text { REFLEXIÓN } \\
+\end{array}$ \\
\hline \multirow{2}{*}{$\begin{array}{c}\text { COGNI- } \\
\text { TIVA }\end{array}$} & A-Intuitiva & in & int + con & con+in & con & Conceptual \\
\hline & B-Desarticulada & $-\mathrm{co}$ & - coto & coto & coto+ot & Compleja \\
\hline \multirow{3}{*}{$\begin{array}{c}\text { METÓ- } \\
\text { DICA }\end{array}$} & C-Ingenua & con:mu & con:inmu & con:huc & con:hucdi & Critica \\
\hline & D-Espontánea & enyer & mem & meconc & meso/va & Metódica \\
\hline & E-Mágica & convma & convautex & convmei & convvame & Cientifica \\
\hline \multirow{3}{*}{$\begin{array}{c}\text { TELEO- } \\
\text { LÓGI- } \\
\text { CA }\end{array}$} & F-Desorientada & $-o b$ & obesm & obesconc & obes+ot & Metaorientada \\
\hline & G-Irresponsable & -cons & consinst & consreg & consglob & Responsable \\
\hline & H-Dependiente & xaut & xposgen & :expcap & pospersvi & Autónoma \\
\hline \multirow{3}{*}{$\begin{array}{l}\text { FOR- } \\
\text { MAL }\end{array}$} & I-Inexpresiva & -regest & estm & estfle & estfleexp & Expresiva \\
\hline & J-Inelegante & -regsissi & resissim & flesissi & flevasissi & Ingeniosa \\
\hline & K-Descontextualizada & comeg & compu+eg & compem & compc & Contextualizada \\
\hline
\end{tabular}

Tabla diseñada por el Dr. Ernesto Sánchez Jerez. Investigador Principal. 
Después de intensa búsqueda encontramos que el trabajo sobre Pedagogía de la Comprensión realizado por Martha Stone y colaboradores, y encontramos niveles de reflexión que nos proporcionaron categorías "vinculadas de modo directo a las características particulares del fenómeno" (Alvarado, Módulo 2). Fueron once categorías en total, cada una con cuatro niveles, como se muestra enseguida y se explicará más adelante.

Las abreviaturas de la tabla corresponden a los siguientes niveles:

A. Intuitiva- Intuición más conocimiento disciplinar - Conocimiento disciplinar con algo de intuición - Conocimiento disciplinar.

B. No hay conexiones - No hay conexión con el todo - Hay conexión con el todo - Hay conexión con el todo y con otras disciplinas.

C. El conocimiento es el mundo -El conocimiento es información sobre el mundo - El conocimiento es humanamente construido - El conocimiento es humanamente construido y discutible.

D. Ensayo y error - Método mecánicamente - Método conscientemente - Método de manera sofisticada y/o varios métodos.

E. Convalida mágicamente - Convalida con autoridad externa - Convalida con método infalible - Convalida con varios métodos.

F. No hay objetivos - Objetivos esenciales mecánicamente - Objetivos esenciales conscientemente - Objetivos esenciales más otros objetivos metadisciplinares.

G. Sin consecuencias-Con consecuencias institucionales - Con consecuencias regionales - Con consecuencias globales.
H. Por autoridad - Por posición general - Como un experto caprichoso - Con posición desde personal visión del mundo.

I. No hay reglas de estilo - Estilo mecánicamente - Estilo usado flexiblemente - Estilo usado flexiblemente y de manera personal.

J. Sin reglas del sistema de signos y símbolos - Reglas del sistema de signos y símbolos usados mecánicamente - Flexibilidad en el sistema de signos y símbolos - Flexibilidad y variedad en el sistema de signos y símbolos.

K. Comunicación egoísta - Comunicación para un público con egoísmo Comunicación para público y contexto mecánicamente - Comunicación para público y contexto

Desde el interaccionismo simbólico, consideramos que el practicante reflexiona en sus intercambios con el usuario, el monitor y el asesor, pues no es un sujeto pasivo sino activo (Blumer, p.2). $Y$ lo hace en un proceso interpretativo que en primera instancia internaliza los significados en un diálogo interior y en segunda, selecciona, evalúa reagrupa y transforma los significados a la luz de la situación y de la dirección de su acción (Blumer, p.5). Los investigadores, analizamos esa reflexión de los practicantes, plasmada en sus diálogos orales y escritos, con la ayuda de las subcategorías que conformaron una tabla con cuarenta y cuatro casillas que corresponden a otros tantos criterios para caracterizarla. Esta caracterización fue un ejercicio interpretativo que no entra en la categoría de las certidumbres matemáticas, sino en el terreno de las probabilidades de las investigaciones cualitativas.

Para elevar el porcentaje de probabilidad se compartieron los resultados de la investigación con los docentes de Dere- 
cho (validez interna) y se compartieron y consensaron significados (Goetz y Lecompte, p.225). Además, se aplicaron las subcategorías utilizadas a la caracterización de la reflexión en las prácticas odontológicas (validez externa, Goetz y Lecompte, p.231) con muy buenos resultados por los menos a nivel de proyecto.

Finalmente se sacaron conclusiones, bastante limitadas, por cierto, dado el carácter idiosincrático de la investigación y el pequeño grupo analizado.

\section{Categorías para Analizar las Prácticas de Consultorio Jurídico}

En nuestra búsqueda por unas categorías que nos apoyen en el trabajo de analizar los textos transcritos de las interven- de la Enseñanza para la ciones de los estudiantes en su labor de práctica del Consultorio Jurídico, encontramos la taxonomía propuesta por Martha Stone en su investi- juicios y transformar la gación sobre la Enseñanza Comprensión propone que el conocimiento se convierte en una herramienta reflexiva para hacer productos, contar historias, resolver problemas, formular vida cotidiana" va de un estudiante, se está analizando su capacidad comprensiva en la acción o el desempeño.

Para David Perkins, del equipo investigador de la autora, la comprensión es "capacidad de desempeño flexible" (Stone, p.70), en donde toda práctica reflexiva es en realidad un "desempeño de comprensión" o "un desempeño comprensivo" (Stone, p.73).

La doctora Stone propone cuatro "dimensiones" para la Comprensión: la Cognitiva, la Metódica, la Teleológica y la Formal. Estas dimensiones tienen en cuenta aspectos presentes en las tradiciones compartidas en una práctica profesional según la referencia de Schön: “Una práctica profesional es la competencia de una comunidad de prácticos que comparten, en palabras de John Dewey, las tradiciones de una profesión.
“Una práctica

profesional es la competencia de una comunidad de prácticos que comparten, en palabras de John Dewey, las tradiciones de una profesión. Comparten convenciones de acción que incluyen medios, lenguajes e instrumentos

distintivos. Funcionan

en el marco de instituciones de un tipo muy particular: por ejemplo, los juzgados, las instituciones educativas, los hospitales y las empresas. Sus prácticas se estructuran en términos de formas particulares de unidades de actividad (por ejemplo: casos, visitas de pacientes o lecciones) y son modeladas a nivel social e institucional de manera que se repiten determinados tipos de situaciones" 
Comparten convenciones de acción que incluyen medios, lenguajes e instrumentos distintivos. Funcionan en el marco de instituciones de un tipo muy particular: por ejemplo, los juzgados, las instituciones educativas, los hospitales y las empresas. Sus prácticas se estructuran en términos de formas particulares de unidades de actividad (por ejemplo: casos, visitas de pacientes o lecciones) y son modeladas a nivel social e institucional de manera que se repiten determinados tipos de situaciones" (SCHÖN, 2002, ps.41-42).

La Dimensión Cognitiva considera cómo en la práctica se transforman las intuiciones en conceptos y teorías y qué redes de conceptos utiliza el practicante (Stone, p.244).

La Dimensión Metódica tiene en cuenta la manera como se utiliza en la práctica un sano escepticismo y cómo se usan los métodos de la propia disciplina (lb.).

La Dimensión Teleológica considera los propósitos del uso del conocimiento en la práctica (lb.).
La Dimensión Formal se lleva a cabo cuando se realiza la práctica con determinados géneros de realización y de sistemas de símbolos (lb.).

Cada uno de estos tipos se subdivide en cuatro niveles: el Ingenuo, el de Novatos, el de Aprendiz y el de Maestría.

En el nivel ingenuo, el practicante utiliza más que nada su intuición (Stone, p.239).

En el nivel de Novatos, utiliza rituales y mecanismos exigidos (Stone, p.240).

En el nivel de Aprendiz, el practicante utiliza con cierta flexibilidad los "conocimientos y modos de pensar disciplinarios" (Stone, p.240).

En el nivel de Maestría se da la integración, la creatividad y la crítica (Stone, p.241).

La reflexión interior del practicante consigo mismo se encuentra frecuentemente en el nivel de Aprendiz, y en la casi totalidad de los procesos del nivel de Maestría.

DIMENSIÓN COGNITIVA²

Tabla 2

\begin{tabular}{|l|l|l|}
\hline \multirow{2}{*}{ Niveles } & \multicolumn{2}{|c|}{ COGNITIVA } \\
\cline { 2 - 3 } & A - Creencias intuitivas transformadas * & B - Redes conceptuales ricas y coherentes** \\
\hline $\begin{array}{l}\text { NIVEL 4 } \\
\text { De maestría }\end{array}$ & Conceptos disciplinarios & $\begin{array}{l}\text { Abstracción extendida: Conexiones con sentido en la } \\
\text { propia área y más alla }\end{array}$ \\
\hline $\begin{array}{l}\text { NIVEL 3 } \\
\text { De aprendiz }\end{array}$ & Conceptos disciplinarios con algunas creencias intuitivas & $\begin{array}{l}\text { Relacional: Muchas conexiones con significación para } \\
\text { el todo }\end{array}$ \\
\hline $\begin{array}{l}\text { NIVEL 2 } \\
\text { De principiante }\end{array}$ & $\begin{array}{l}\text { Creencias intuitivas con algunos conocimientos disci- } \\
\text { plinarios }\end{array}$ & $\begin{array}{l}\text { Multiestructural: Muchas conexiones sin metaconexio- } \\
\text { nes ni significación para el todo }\end{array}$ \\
\hline $\begin{array}{l}\text { NIVEL 1 } \\
\text { Ingenuo }\end{array}$ & Creencias intuitivas, folclóricas o míticas & Uniestructural: Conexiones obvias sin significación \\
\hline
\end{tabular}

2 Los "niveles y dimensiones" son expuestos por Martha Stone en su trabajo; igualmente el contenido de las "Creencias intuitivas transformadas". El contenido de los niveles de "Redes conceptuales ricas y coherentes" es tomado de la taxonomía SOLO (Structure of Observed Learning Outcomes), desarrollada por Biggs y Collis en 1982, pues creemos que los niveles de Stone coinciden casi exactamente con los niveles propuestos por aquellos. 


\section{Recolección y Análisis De Datos}

\section{EL CONTEXTO}

La facultad de Derecho y Ciencias políticas comenzó con la seccional de la USTA en Bucaramanga, en 1973. Inició con 146 estudiantes. Fue una réplica de la facultad de Derecho de Bogotá, inclusive en el sistema Modular, propio de esta carrera ${ }^{3}$.

Como parte de la formación profesional en Derecho de la Universidad Santo Tomás, los estudiantes en sus últimos años realizan prácticas con casos reales en el denominado Consultorio Jurídico. Éste comienza a funcionar en 1977. Dada la importancia concedida por las directivas a este aprendizaje por la práctica, actualmente los estudiantes realizan Consultorio Jurídico durante cuatro semestres: VII, VIII, IX y X (Ver historia del Consultorio en el Anexo 5, en una investigación de la Doctora Ángela Tarazona).

Según el Reglamento del Consultorio Jurídico de la USTA de Bucaramanga, éste tiene como objetivo académico "capacitar a los alumnos para el ejercicio profesional" y como objetivo de proyección social "brindar asesoría jurídica, judicial y extrajudicial, en forma totalmente gratuita a las personas de escasos recursos económicos" (Reglamento, art. 2).

El consultorio cuenta con asesores especializados y experimentados para tratar los problemas de los usuarios en "Derecho Público, Derecho Penal, Derecho Privado y Derecho Laboral " (art. 7). Igualmente cuenta con Monitores, que son abogados recién egresados,

3 "Por eso no extrañará que los primeros programas, los llamados 'analíticos', fueran idénticos con los de Bogotá. Las modificaciones que experimentaban los de Bogotá, regían igualmente para los de Bucaramanga. Así, cuando se impuso en Bogotá el denominado 'Sistema modular', de inmediato se trasladaron sus personajes, autores, para ilustrar al profesorado de Bucaramanga e implantar el nuevo sistema. Hoy se practica, si bien ha experimentado diversas transformaciones con el correr del tiempo" (Gutiérrez, 2003, p.47). que resuelven, en primera instancia, las dudas de los practicantes, y a quienes los estudiantes deberán pasarle un informe semanal "sobre cada una de sus actuaciones en los casos a su cargo " (art. 19).

Las interacciones en el consultorio jurídico son de diversa índole. Se realizan entre los monitores y los usuarios, entre los practicantes y los usuarios, entre los practicantes y los monitores, y entre los practicantes y los asesores. El proceso seguido en el consultorio se inicia con la recepción del caso por parte del Monitor asignado, «quien se cerciorará sobre la clase de negocio de que se trata, de la competencia del Consultorio Jurídico y de la capacidad económica del solicitante» (art. 20). Este mismo Monitor asigna el caso a uno de los alumnos practicantes, quien interactúa directamente con los usuarios. El estudiante hará una entrevista al usuario en los formatos especiales del Consultorio (Apéndice 2). Luego se dan las interacciones entre los estudiantes y los monitores. «Recaudados la totalidad de los datos exigidos, el alumno presentará al monitor la entrevista para su revisión, éste podrá entonces hacer todas las observaciones que estime pertinentes y exigir el recaudo de datos adicionales si lo considera necesario. La entrevista concluirá con la firma del usuario, del alumno entrevistado y del monitor» (art. 20).

El caso se repartirá entre los estudiantes practicantes. El alumno responsable del negocio tendrá: «Tres (3) días hábiles para emitir su concepto jurídico o elaborar y entregar poderes, o contestar demandas civiles, laborales y aquellas de familia que puedan ser elaboradas a nombre del usuario del servicio; cinco (5) días hábiles para elaborar y entregar liquidaciones y ocho (8) días hábiles para la elaboración y presentación de toda demanda» (art. 24).

En el curso del proceso, el practicante sigue interactuando con el usuario, con el Monitor y también con el Asesor. El estudiante atenderá a los usuarios cumpli- 
damente con las citaciones que acuerde con el mismo. Igualmente deberá «cumplir con los términos para la elaboración, revisión por Monitor, revisión por Asesor y entrega del trabajo asignado" (art. 16,f). Además llevará una especie de bitácora en donde anotará las fechas y todo lo que suceda en el negocio a su cargo ${ }^{4}$. Semanalmente informará al Monitor sobre sus actuaciones y contará con la revisión del mismo y del Asesor para sus actuaciones ante juzgados o para el usuario (art. $16, p)$. Igualmente informará al usuario sobre sus actividades relacionadas con el negocio cuando éste lo requiera, y le enviará dos veces por semestre un informe escrito de las mismas (art. 16,0).

Una vez finalizado el negocio, el estudiante presentará antes de cinco días, solicitud de archivo del negocio (art.29).

\section{TRABAJO DE CAMPO}

Se realizó el trabajo de campo el segundo semestre de 2008, en el Consultorio Jurídico de la carrera diecinueve con calle novena. Éste fue escogido por la cercanía a la Sede Central de la Universidad, en donde se desarrolla nuestra labor docente, y porque allí se asesoran muchos procesos en todas las áreas del derecho. Se hicieron varias actividades:

Primero. Se exploró el ambiente jurídico del Consultorio, releyendo el Reglamento seguido por los estudiantes, asistiendo al sitio, observando su movimiento y los pasos seguidos por los practicantes. Con la Directora del Consultorio y con dos Monitores analizamos qué casos podrían ser estudiados, y se decidió descartar aquellos que son meramente mecánicos, como el cálculo de unas prestaciones, y

\footnotetext{
4 El artículo16 del reglamento del Consultoro jurídico dice al respecto: "g. Mantener debidamente actualizado el cartón de control de cada caso asignado, anotando con exactitud cronológicamente todas las actuaciones realizadas, las novedades que se presenten, así como la conclusión a que se llegó en cada uno de los asuntos a él encomendados; de igual manera, deberá anotarse si el caso pasó a un monitor por razón de competencia ».
}

dedicarnos a los que tenían alguna complejidad jurídica. Se contó con el apoyo de todos los Practicantes y de todos los Monitores y Asesores. Se trataba, entonces, de acceder a los procesos más o menos complejos que estuvieran en curso.

El Consultorio trabaja cuatro ramas del Derecho: el Civil, el Público, el Laboral y el Penal. Además realiza Conciliaciones. Entonces escogimos casos que estuvieran en curso y que representaran cada una de esas ramas, cinco en total, más las Conciliaciones.

Segundo. Se filmó las monitorías y asesorías de los casos y las entrevistas realizadas con los practicantes. Es de notar la colaboración de los Practicantes, Monitores y Asesores, así como de la doctora Matilde. Se hizo lo siguiente:

\section{- Derecho Civil:}

Sobre el concepto jurídico, en un tema de Bienes públicos, sobre un terreno Baldío, se filmó:

-la monitoría,

-la asesoría,

-dos entrevistas con la practicante.

Sobre la tramitación de un proceso ejecutivo singular en condición de demandado, filmamos:

-la asesoría,

-una entrevista con la practicante.

\section{- Derecho Laboral:}

Sobre el caso de la reclamación a una cooperativa de trabajo, filmamos:

-una larga entrevista con el practicante.

\section{- Derecho Penal:}

Sobre el robo de una moto, se filmó: 
-la monitoría,

-la asesoría,

-una entrevista con el practicante.

\section{- Conciliación:}

Sobre una audiencia para ejecución de obligación alimentaria, se filmó:

-el análisis de la Conciliación, realizado la Directora, el Monitor y el Practicante,

-una entrevista con el practicante.

Tercero. Se fotocopió los documentos de los distintos casos. Estos aparecen sin nombres, por la reserva jurídica obligatoria para los procesos, especialmente para los Ilevados por los Consultorios Jurídicos.

Cuarto. Se seleccionó los casos que contaban con Monitoría y Asesoría. Fueron tres: El del Concepto jurídico sobre un baldío, el de Penal y el de Laboral. De este último no obtuvimos filmaciones de la Monitoría ni de la asesoría, pero obtuvimos las revisiones escritas hechas a los textos presentados por el Practicante. Del Concepto jurídico no se tuvo en cuenta la Monitoría ni la Asesoría orales pues éstas sólo hacían referencia a los textos escritos.

\section{EL CONSULTORIO JURÍDICO COMO PRÁCTICUM REFLEXIVO}

Las prácticas del Consultorio jurídico constituyen verdaderas intervenciones sobre la realidad legal de los usuarios. Sin embargo, en el espacio entre la recogida de datos de los usuarios y la aplicación a la realidad jurídica de los mismos, se da un aprender a través de la realización de proyectos que no llegan directamente a la realidad sino que parecen simularla y simplificarla (Schön, 1987, p.46) gracias a que las consecuencias de lo hecho no afectan a nadie sino que son absorbidas y modificadas por los tutores. Esto es, se configuran Prácticum reflexivos: los proyectos de los practicantes son diseños tentativos que son revisados por los Monitores y Asesores, reelaborados y sólo cuando llenan todos los requisitos jurídicos pasan a las instancias reales. Están constituidos por una etapa de diseño, otra de tutoría y otra de rediseño al menos.

Veamos cómo ocurre este interregno reflexivo en los tres casos:

\section{a- REALIDAD}

-El practicante del Concepto jurídico recoge los datos del mismo usuario.

-El practicante de Laboral recoge los datos sobre un caso en el que se presentó la demanda, se recogieron los testimonios y entra a etapa de alegatos de conclusión.

-El practicante de Penal es designado por un juez como defensor de oficio en un caso de hurto de una moto.

\section{b- DISEÑO}

-El practicante del Concepto jurídico obtiene orientación del Monitor, elabora problemas y los soluciona por escrito.

-El practicante de Laboral, orientado por lo realizado por compañeros que lo precedieron en el caso, se construye interrogantes que resuelve por escrito en alegatos de conclusión.

-El practicante de Penal, orientado por las características del caso, revisa la literatura legal y procesal al respecto para participar en la Audiencia de legalización de captura y posteriormente en la Audiencia de verificación de allanamiento y fallo.

$$
\text { c- TUTORÍA }
$$

-Los practicantes de Civil y Laboral reciben tutoría de sus respectivos Monitores quienes hacen observaciones y correcciones por escrito. Al final interviene el Asesor. 
-El practicante de Penal recibe tutoría oral del Monitor y el Asesor para prepararse para las respectivas Audiencias.

\section{d- REDISEÑO}

-Los practicantes de Civil y Laboral reelaboran dos veces sus escritos jurídicos.

-El practicante de Penal reelabora su estrategia con la ayuda del Asesor, sobre todo.

\section{e- APLICACIÓN}

-Cuando los escritos de Civil y Laboral están corregidos debidamente se presentan a las respectivas instancias jurídicas.

-Con la preparación adecuada el practicante de Penal afronta las Audiencias de legalización de captura y de verificación de allanamiento y fallo.

\section{4-ANÁLISIS CATEGORIAL}

\begin{tabular}{|l|l|c|c|}
\hline RAMA JURÍDICA & DOCUMENTO & TABLAS DE TEXTO & RESÚMENES \\
\hline & -Entrevista a usuario & 2 & 0 \\
& -Primer concepto del practicante & 7 & 0 \\
DERECHO CIVIL & -Revisión del Monitor & 7 & 0 \\
& -Segundo concepto del practicante & 4 & $4 \mathrm{t}$ \\
& -Entrevista con investigador uno & 1 & $1 \mathrm{t}$ \\
\hline & -Entrevista con investigador dos & 1 & 1 \\
DERECHO PENAL & -Informe actuaciones realizadas & 1 & 1 \\
& -Interacción con Monitor & 1 & 1 \\
\hline & -Interacción con Asesor & 1 & $1 \mathrm{t}$ \\
DERECHO LABORAL & -Entrevista con investigador & 1 & 0 \\
& -Primeros alegatos de conclusión & 1 & $1 \mathrm{t}$ \\
\hline TOTAL & - Segundos alegatos de conclusión & 0 & 12 \\
\hline
\end{tabular}

Recogimos 14 documentos, entre textos, interacciones y entrevistas:

Tabla No. 13

Cada uno de los 14 documentos fue transcrito a una tabla de texto, como la siguiente, para poder identificar las categorías de reflexión y su respectivo nivel.

\begin{tabular}{|c|c|c|c|c|c|c|c|c|c|c|c|c|}
\hline \multicolumn{11}{|c|}{ CATEGORÍA } & SUBCATEGOR & TRANSCRIPCIÓN ESCRITA \\
\hline A & B & c & D & $\mathrm{E}$ & $\mathrm{F}$ & G & $\mathrm{H}$ & I & $\mathrm{J}$ & $\mathrm{K}$ & & \\
\hline A & B & c & D & $\mathrm{E}$ & $\mathrm{F}$ & G & $\mathrm{H}$ & 1 & $\mathrm{~J}$ & $\mathrm{~K}$ & & \\
\hline 4 & 3 & 2 & 3 & 3 & 3 & 4 & 3 & 3 & 3 & 4 & & \\
\hline
\end{tabular}

Tabla No. 14

\section{RESULTADOS}

Al hacer un recuento de los análisis anteriores se obtuvo los siguientes resultados:

\section{A-CASO BALDÍO}

Los investigadores estudian las dos versiones del Concepto jurídico presentado por el practicante y le realizan dos entrevistas. 


\section{1- CONCEPTO ESCRITO}

En la construcción del Concepto se trabajan cuatro elementos: los hechos, el problema, los fundamentos jurídicos y la conclusión. La diferencia entre la primera versión (oscuro) y la segunda (M) aparecen en la siguiente tabla.

CATEGORÍAS PROMEDIADAS DE CONCEPTO JURÍDICO UNO Y DOS DE PRACTICANTE

\begin{tabular}{|c|c|c|c|c|c|}
\hline REFLEXIÓN & Niv.1 & Niv.2 & Niv.3 & Niv.4 & REFLEXIÓN \\
\hline Intuitiva & & & MMM & MMM & Conceptual \\
\hline Desarticulada & & & MMM & & Compleja \\
\hline Ingenua & & MMM & MMM & MMM & Crítica \\
\hline Espontánea & & MMM & MMM & & Metódica \\
\hline Mágica & & & MMM & & Científica \\
\hline Desorientada & & & MMM & & Metaorientada \\
\hline Irresponsable & & MMM & MMM & MMM & Responsable \\
\hline Dependiente & & MMM & MMM & & Autónoma \\
\hline Inexpresiva & & MMM & MMM & & Expresiva \\
\hline Inelegante & & MMM & & & Ingeniosa \\
\hline Descontextualizada & & MMM & MMM & & Contextualizada \\
\hline
\end{tabular}

Tabla 45

-Es notable la influencia del Monitor en el crecimiento cognitivo y metodológico del practicante.

-En la dimensión cognitiva, el practicante pasa de utilizar creencias intuitivas a usar conceptos de su disciplina jurídica. También, de conexiones sin conexión con el todo, a relaciones conceptuales con sentido para el Concepto jurídico sobre la adquisición de un baldío.

-El practicante aprende a considerar las leyes y los procedimientos como construidos por el hombre y criticables, y no como reglas estáticas y perennes. Usa conscientemente el método exegético deductivo, con la ayuda del Monitor, para manejar y convalidar el conocimiento.

-El practicante logra identificar y activar las finalidades del Derecho, de los baldíos y de la economía, hasta llegar a tener cierta libertad para establecer sus propias metas en la consecución del baldío para el usuario. Tiene en cuenta las consecuencias del concepto tanto para el usuario como para las instancias jurídicas.

-El practicante usa un estilo convincente pero pesado por el exceso de citas textuales. Tiene dificultades con algunas expresiones gramaticales y con un manejo un poco descuidado de la revisión del texto. Sin embargo trata de tener en cuenta los intereses y necesidades del usuario y del respaldo jurisprudencial del concepto.

\section{2- ENTREVISTAS}

La siguiente tabla muestra el estado de las categorías reflexivas en la entrevista realizada al practicante. 
CATEGORÍAS DE ENTREVISTA PRIMER INVESTIGADOR

\begin{tabular}{|l|l|l|l|l|l|}
\hline REFLEXIÓN & Niv.1 & Niv.2 & Niv.3 & Niv.4 & REFLEXIÓN \\
\hline Intuitiva & & & & & Conceptual \\
\hline Desarticulada & & & & & Compleja \\
\hline Ingenua & & & & & Crítica \\
\hline Espontánea & & & & & Metódica \\
\hline Mágica & & & & & Científica \\
\hline Desorientada & & & & & Metaorientada \\
\hline Irresponsable & & & & & Responsable \\
\hline Dependiente & & & & & Autónoma \\
\hline Inexpresiva & & & & & Expresiva \\
\hline Inelegante & & & & & \\
\hline Descontextualizada & & & & & Contextualizada \\
\hline
\end{tabular}

Tabla 46

-El practicante se desenvuelve bien a nivel oral y muestra una gran capacidad de comprensión y una gran sensibilidad hacia el entrevistador.

-En la dimensión cognitiva expresa manejo de conceptos jurídicos y elabora relaciones con sentido para el conjunto del Concepto.

-Utiliza el método exegético para exponer sus conceptos jurídicos y para convalidar el conocimiento. Por la corrección de Monitor descubre la relatividad de las normas y de los decretos reglamentarios.

-Conoce los objetivos de la adjudicación de un baldío y los compromisos éticos. Dirige su esfuerzo a proporcionar consecuencias favorables para el usuario, aunque todavía su posición personal depende mucho de sus asesores.

-Expresa gran contextualización al escuchar al entrevistador y al adaptar su lenguaje al de él. Sin embargo, usa algunas expresiones incorrectas. Acostumbra autopreguntarse para aclarar su pensamiento y su expresividad.

-La ética forma parte de su preocupación y el deseo de respetar a la persona.

-Se desempeña mejor oralmente que por escrito.

\section{B- CASO PENAL}

En el caso penal por hurto calificado, el practicante expresa los siguientes niveles de reflexión:

CATEGORÍAS DE INTERACCIONES CON EL MONITOR Y EL ASESOR

\begin{tabular}{|l|c|c|c|c|c|}
\hline REFLEXIÓN & Niv.1 & Niv.2 & Niv.3 & Niv.4 & REFLEXIÓN \\
\hline Intuitiva & $\mathrm{A}$ & $\mathrm{A}$ & $\mathrm{A}$ & $\mathrm{A}$ & Conceptual \\
\hline Desarticulada & $\mathrm{A}$ & $\mathrm{A}$ & $\mathrm{A}$ & $\mathrm{A}$ & Compleja \\
\hline Ingenua & $\mathrm{A}$ & $\mathrm{A}$ & $\mathrm{A}$ & & Crítica \\
\hline Espontánea & $\mathrm{A}$ & $\mathrm{A}$ & $\mathrm{A}$ & & Metódica \\
\hline Mágica & $\mathrm{A}$ & $\mathrm{A}$ & $\mathrm{A}$ & $\mathrm{A}$ & Científica \\
\hline Desorientada & $\mathrm{A}$ & $\mathrm{A}$ & $\mathrm{A}$ & $\mathrm{A}$ & Metaorientada \\
\hline Irresponsable & $\mathrm{A}$ & $\mathrm{A}$ & $\mathrm{A}$ & & Responsable \\
\hline Dependiente & $\mathrm{A}$ & $\mathrm{A}$ & & & Autónoma \\
\hline Inexpresiva & $\mathrm{A}$ & $\mathrm{A}$ & $\mathrm{A}$ & & Expresiva \\
\hline Inelegante & $\mathrm{A}$ & $\mathrm{A}$ & $\mathrm{A}$ & & Ingeniosa \\
\hline Descontextualizada & $\mathrm{A}$ & $\mathrm{A}$ & $\mathrm{A}$ & $\mathrm{A}$ & Contextualizada \\
\hline
\end{tabular}

Tabla 47 
Las casillas oscuras corresponden a la interacción con el Monitor y las que tienen A, la interacción con el Asesor.

-Es importante el trabajo realizado por el Monitor y el Asesor para reflexionar sobre su estrategia argumentativa. Gracias a ello y a las capacidades cognitivas del estudiante, su nivel de reflexión es bastante alto.

-En la dimensión cognitiva utiliza conceptos jurídicos correctamente y hace conexiones que tienen que ver con el caso de robo. El Asesor ayuda a elevar el nivel a relaciones complejas.

-Ejecuta el método de la Práctica Reflexiva de diseño-acción-rediseño, con la ayuda del Monitor y el Asesor en circunstancias en las que debe actuar solo. Para su actuación como defensor de oficio utiliza el método exegético y el de la buena fe, que a veces en la práctica no funcionan. Sin embargo, con el Asesor pierde un poco su independencia.
-Se preocupa por los intereses del usuario con mucha dedicación y empatía. Tiene en cuenta los objetivos del proceso y de la ética y actúa con relativa independencia. Manifiesta una posición crítica frente a la administración de la justicia en Colombia.

-Es fluido en su expresión y sabe escuchar y adaptar su vocabulario a las capacidades del entrevistador y del usuario.

-La práctica reflexiva hace surgir lo mejor de este practicante y le devela sus pequeñas falencias.

\section{C-CASO LABORAL}

Los investigadores analizan los alegatos que el practicante presenta por escrito al Monitor y al Asesor, y la entrevista que le hicieron en las instalaciones del Consultorio Jurídico de Bucaramanga.

\section{1-ALEGATOS ESCRITOS}

Analizando la tabla siguiente, que presenta las revisiones de los textos uno (oscuro) y dos (M) de alegatos de conclusión, podemos llegar a varias conclusiones.

CATEGORÍAS DE DOCUMENTO DOS DE ALEGATOS DE CONCLUSIÓN

\begin{tabular}{|l|l|l|l|l|l|}
\hline REFLEXIÓN & Niv.1 & Niv.2 & Niv.3 & Niv.4 & REFLEXIÓN \\
\hline Intuitiva & & & MMM & MMM & Conceptual \\
\hline Desarticulada & & & MMM & & Compleja \\
\hline Ingenua & & & MMM & MMM & Crítica \\
\hline Espontánea & & & MMM & MMM & Metódica \\
\hline Mágica & & & MMM & MMM & Científica \\
\hline Desorientada & & & MMM & & Metaorientada \\
\hline Irresponsable & & & MMM & & Responsable \\
\hline Dependiente & & & MMM & & Autónoma \\
\hline Inexpresiva & & & MMM & & Expresiva \\
\hline Inelegante & & & & & Ingeniosa \\
\hline Descontextualizada & & & MMM & & Contextualizada \\
\hline
\end{tabular}

Tabla 48

-En primer lugar, la influencia decisiva del Monitor en el progreso del practicante en su proceso reflexivo. Al comenzar, el practicante se encuentra en el segundo nivel en casi todos los aspectos de la reflexión; con la ayuda del Monitor, quien le colabora en ésta, asciende por lo menos un nivel en casi todos los ítems.
-En la dimensión cognitiva, la reflexión del estudiante pasa de un manejo bastante intuitivo del caso a una reflexión en donde se imponen los conceptos jurídicos de manera sobresaliente. Por otro lado, las redes cognitivas que elabora tienen sentido para y desde el todo; aunque no sale 
del campo jurídico a otros terrenos de la realidad del usuario y de las Cooperativas.

-En la dimensión metódica es donde se dan más avances en el practicante. De una reflexión ingenua, asciende a otra con conciencia de que los seres humanos construimos todo el conocimiento: el teórico, el procedimental y el perceptivo. De un método aplicado mecánicamente, pasan al uso de varios métodos en la solución de su caso. De una convalidación por autoridad externa, sube al nivel más alto, en donde utiliza varios métodos científicos de la propia disciplina. En su manera de investigar, el estudiante comienza con un método analógico que lo guía un poco por fuera de los procedimientos eficaces; pero esto es corregido por el Monitor. En su método expositivo, él quiere argumentar basándose equivocadamente en el recuento del proceso realizado, pero gracias al Monitor se encamina por la vía de la prueba testimonial y el argumento jurídico y jurisprudencial.

-En la dimensión teleológica el practicante inicia con un buen nivel en la consideración de las consecuencias. Hay un alto nivel de conciencia de las consecuencias de sus acciones jurídicas con relación, sobre todo, a los intereses del usuario, gracias a una relación empática y solidaria con el mismo. Con relación a los objetivos que orientan el trabajo jurídico, hay una gran conciencia de los mismos, pero hay una incapacidad metodológica de hacer lo necesario para alcanzarlos, que es superada con la ayuda del Monitor. Con relación a la autonomía en las metas del trabajo, pasa de una dependencia de los modelos, a un espacio más amplio abierto por el Monitor, en donde surge el propósito de convencer al juez con argumentos empírico legales y jurisprudenciales.

-La dimensión formal es la que sufre menos transformaciones por parte del practicante, sobre todo en la elegancia e ingenio en el manejo del lenguaje escrito. Parece que hay un poco de descuido en este aspecto y no aparece el sentido de la autocrítica y la autocorrección, pues al final aparecen los mismos problemas de tildes, de concordancia de género y de faltas ortográficas; el Monitor no tiene tiempo para tratar concienzudamente este aspecto. En su tercer escrito, el practicante mejora su estilo de presentación de los argumentos y expresa mayor conciencia de los intereses del juez y del usuario; con relación a éste último establece una empatía que lo lleva a escribir con cierta pasión muy conveniente en estos casos.

\section{2-ENTREVISTA}

Al analizar la tabla categorial de la entrevista realizada por los dos investigadores al practicante, se puede observar lo siguiente:

CATEGORÍAS DE ENTREVISTA INVESTIGADORES UNO Y DOS ALEGATOS DE CONCLUSIÓN

\begin{tabular}{|l|l|l|l|l|l|}
\hline REFLEXIÓN & Niv.1 & Niv.2 & Niv.3 & Niv.4 & REFLEXIÓN \\
\hline Intuitiva & & & & & Conceptual \\
\hline Desarticulada & & & & & Compleja \\
\hline Ingenua & & & & & Crítica \\
\hline Espontánea & & & & & Metódica \\
\hline Mágica & & & & & Científica \\
\hline Desorientada & & & & & Metaorientada \\
\hline Irresponsable & & & & & Responsable \\
\hline Dependiente & & & & & Autónoma \\
\hline Inexpresiva & & & & & Expresiva \\
\hline Inelegante & & & & & Ingeniosa \\
\hline Descontextualizada & & & & & Contextualizada \\
\hline
\end{tabular}

Tabla 49 
-Es notoria la capacidad oral del practicante, sus recursos, su interés y su optimismo con respecto al caso de los alegatos.

-En lo cognitivo, el estudiante utiliza conceptos adecuados del campo jurídico. Además hace relaciones conceptuales referidas coherentemente al caso de los alegatos.

-En la dimensión metódica, evidencia su percepción de que el conocimiento es una construcción humana que puede fallar, no solo en su propio trabajo, sino en los datos procedimentales recogidos. Para su construir y convalidar conocimiento utiliza el método de la práctica reflexiva, que actúa en la espiral ascendente diseño-acción-rediseño; aunque inicialmente parecía de tipo deductivo, como en la racionalidad técnica, pues se refería a los alegatos como "un caso típico de Cooperativas de Trabajo Asociado", al comenzar actuó como un novato "en un mundo desconocido"; realizó su diseño, elaboró los alegatos y debió rediseñarlos con la reflexión colaborativa del Monitor para rehacerlos de nuevo. Este método de reflexión en la práctica fue privilegiado por el mismo practicante al reconocer el origen del saber en su propia práctica con las cooperativas y en la experiencia del Asesor. En este método es importante la relación afectiva con el usuario, que él expresa al considerar paisana a la usuaria.

-El practicante tiene claras sus finalidades, considerando no solo las del caso, sino las éticas, las de las cooperativas y las del Monitor y el Asesor. Tiene presente las consecuencias de su acción en los alegatos y exhibe un motivador optimismo. Los objetivos del caso se vuelven como un objetivo personal que lo llevan a interesarse en el mismo y a trabajar con bastante pasión y convicción.

-Es notable el aspecto formal de su entrevista. Maneja un estilo claro, ameno y convincente utilizando recursos que evidencian cualidades histriónicas. Es susceptible a los intereses del usuario y el juez, pero también a los de los investigadores. Responden con claridad y pedagogía a las preguntas de los investigadores, que no son expertos en Derecho. Presenta muy pocos errores de construcción gramatical.

-Al comparar los resultados de la entrevista y de los alegatos escritos, se evidencia un mejor desempeño en la expresión oral.

-En ambos se ve clara la influencia positiva del Monitor, que los lleva a reflexionar sobre su trabajo y aceptar sus propios errores con el fin de corregirlos.

-Es satisfactorio constatar la presencia de la ética en la construcción de los alegatos y la importancia que le atribuyen para el éxito profesional. Es notorio el respeto por la persona en su intencionalidad y en su trato personal con el usuario y con el Monitor y el Asesor.

\section{Los Practicantes}

Los Practicantes fueron estudiantes de décimo semestre, que realizaban su segunda práctica de Consultorio Jurídico.

Los investigadores se preguntan si hubo alguna correlación entre los comienzos de los Practicantes y las notas de los distintos semestres. Porque, al parecer, los comienzos del Practicante de Laboral y de Civil fueron bastante deficientes, y fueron ascendiendo en su nivel de reflexión hasta llegar a niveles meritorios después de sus interacciones sobre todo con los Monitores. En cambio, el comienzo del Practicante de Penal fue meritorio y se mantuvo en su nivel todo el tiempo.

Se encontró que el nivel académico de los tres era bastante similar. Los tres hicieron su carrera nivelados, sin perder ningún semestre. Los tres estaban graduados de Abogados en el 2009.

Pero, al parecer, las notas en las clínicas y asignaturas teóricas de sus áreas de 
Derecho no corresponden con su desempeño en las prácticas respectivaa. Por ejemplo, las notas en Clínica de Derecho Laboral son mejores para los practicantes de Derecho Penal y Civil, siendo la peor para el practicante de Laboral. Las mejores notas en Derecho Penal fueron para el practicante de Laboral, mientras que el practicante de Penal siempre ocupó un segundo lugar. Las notas en clínica de Civil fueron mejores para el practicante de Derecho penal, mientras que los practicantes de Laboral y Civil ocuparon un segundo lugar.

\section{Conclusiones}

Las conclusiones que surgen de nuestra investigación tienen un alcance muy limitado, ya que sólo se refieren a tres casos considerados. Sin embargo, son un síntoma que puede ser estudiado más exhaustivamente y son una provocación para otras investigaciones y para acciones metodológicas alternativas.

\section{Estas son:}

-La práctica del Consultorio Jurídico es un verdadero Prácticum Reflexivo con todas las dificultades y bondades del mismo.

-La relación entre docente y practicante presenta un nivel de igualdad y compañerismo en el Consultorio, que bien podría ser asumida en otras instancias pedagógicas de la Facultad de Derecho y de la Universidad.

-No hay duda que la Práctica Reflexiva del Consultorio Jurídico eleva el nivel de reflexión de los estudiantes en las dimensiones cognitiva, metódica y teleológica.

-El buen desempeño de los estudiantes a nivel teórico no garantiza su buen desempeño a nivel práctico.

-Los conocimientos adquieren significado para los practicantes en la medida en que los relacionan con el caso concreto.
-Los Monitores son los que más influyen en el crecimiento de los niveles de reflexión del estudiante y desempeñan una función esencial en el proceso de la Práctica Reflexiva.

-Los objetivos éticos son vivenciados por los estudiantes en su interacción con el usuario.

-La expresión escrita es todavía deficiente y amerita un mayor esfuerzo.

-Un diseño metodológico tipo Prácticum Reflexivo en cada asignatura puede ser una alternativa pedagógica que mejore el desempeño práctico del futuro profesional.

\section{Propuesta Metodológica}

Después de analizar la eficacia pedagógica en los tres practicantes del Consultorio Jurídico de distintas ramas del Derecho, se elaboró, fundamentados en el método del Consultorio y en las teorías estudiadas, el siguiente modelo de Prácticum reflexivo.

\section{El Prácticum Reflexivo}

Con base en la teoría de Schön, emerge una primera estructura con estos pasos: -HECHOS, -ENCUADRE, -PROBLEMAS, -SOLUCIONES (CON EXPERIMENTOS). El encuadre es el marco referencial que orienta (Schön, 1983, p.47) toda la labor de procesamiento de los hechos, desde el planteamiento de los problemas hasta las soluciones. Se plantean las soluciones, pero si no son satisfactorias se dan experimentos mentales que pueden plasmarse en dibujos o en palabras con consecuencias evaluables, a partir de nuevos encuadres (Schön, 1983, p.67). La reflexión está presente en todos los pasos (lb.).

Posteriormente, en la construcción de Categorías de reflexión para la práctica del Consultorio recurrimos a los trabajos de Martha Stone y de David Perkins so- 
bre Pedagogía para la Comprensión. De aquí surgen cuatro dimensiones para la
Reflexión: la Cognitiva, la Metodológica, la Teleológica y la Formal.

Tabla 50. Dimensiones de Reflexión

\begin{tabular}{|l|l|}
\hline TELEOLÓGICA & $\begin{array}{l}\text { Conjunto de “propósitos e intereses que orientan la construcción del conocimiento" (Boix y } \\
\text { Gardner en Stone, p.235). }\end{array}$ \\
\hline METÓDOLÓGICA & $\begin{array}{l}\text { Constituída por un sano escepticismo y por el “uso de métodos confiables para construir y validar } \\
\text { afirmaciones y trabajos verdaderos” (Ib. 232). }\end{array}$ \\
\hline COGNITIVA & Conjunto de creencias, saberes, teorías y saberes con diverso grado de integración (Ib.230-231). \\
\hline FORMAL & Conjunto de géneros de comunicación y sistemas de símbolos usados para comunicarse (lb. 237). \\
\hline
\end{tabular}

Estas Dimensiones se dividen a su vez en once Categorías de reflexión, como se muestra en la tabla siguiente. Se pre- sentan los niveles inferior y superior de reflexión.

Tabla 51. Categorías de Reflexión

\begin{tabular}{|l|l|l|}
\hline DIMENSIÓN & REFLEXIÓN - & REFLEXIÓN + \\
\hline \multirow{4}{*}{ TELEOLÓGICA } & Desorientada & Metaorientada \\
\cline { 2 - 3 } & Irresponsable & Responsable \\
\cline { 2 - 3 } & Dependiente & Autónoma \\
\hline \multirow{4}{*}{ METÓDOLÓGICA } & Ingenua & Crítica \\
\cline { 2 - 3 } & Espontánea & Metódica \\
\cline { 2 - 3 } & Mágica & Científica \\
\hline \multirow{3}{*}{ FOGNITIVA } & Intuitiva & Conceptual \\
\cline { 2 - 3 } & Desarticulada & Compleja \\
\hline \multirow{3}{*}{ FORMAL } & Inexpresiva & Expresiva \\
\cline { 2 - 3 } & Inelegante & Ingeniosa \\
\cline { 2 - 3 } & Descontextualizada & Contextualizada \\
\hline
\end{tabular}

La reflexión metaorientada plantea propósitos que van más allá de la propia disciplina, visualizando valores generales y horizontes transdisciplinarios.

La responsable considera las consecuencias inmediatas y mediatas del objeto de análisis y de los aportes planteados.

La autónoma expresa propósitos personales que nazcan del propio convencimiento.

La reflexión crítica adopta un sano escepticismo como método permanente.
La metódica, cuando se procede siguiendo uno o varios métodos de reconocida validez.

La científica, cuando utiliza métodos vigentes en las búsquedas de los científicos.

La reflexión conceptual utiliza conceptos propios de la disciplina y no se reduce a lugares comunes o saberes intuitivos.

La compleja hace relaciones con conceptos intra y extradisciplinares. 
La reflexión expresiva es la que utiliza géneros adecuados para comunicar la disciplina.

La ingeniosa, cuando usa figuras, metáforas y términos que muestran un notable manejo de los signos y símbolos.

y la contextualizada, cuando tiene en cuenta el público destinatario y las circunstancias apropiadas.

Con el aporte de la Pedagogía de la Comprensión, el Prácticum Reflexivo queda estructurado de este modo:

A. HECHOS, tomados de la realidad;

B. PROPÓSITOS, que encuadran todo el resto del proceso, preferiblemente desde una reflexión metaorientada, responsable y autónoma;

C. PROBLEMAS, que no existen en la realidad, sino que son creados por los profesionales desde el horizonte de los propósitos (Schön, 1983, p.26);

D. MÉTODOS, para buscar contenidos y para construir las soluciones con reflexión realmente metódica, científica y crítica;

E. FORMA, o manera de comunicar el proceso a los demás desde una reflexión expresiva, ingeniosa y contextualizada;

F. CONTENIDOS, constituidos por saberes, reflexionados conceptualmente y de manera compleja;

G. SOLUCIONES, al problema o problemas presentados;

H. ANALOGÍAS, o problemas parecidos con soluciones parecidas.

Tabla 52. El Prácticum Reflexivo

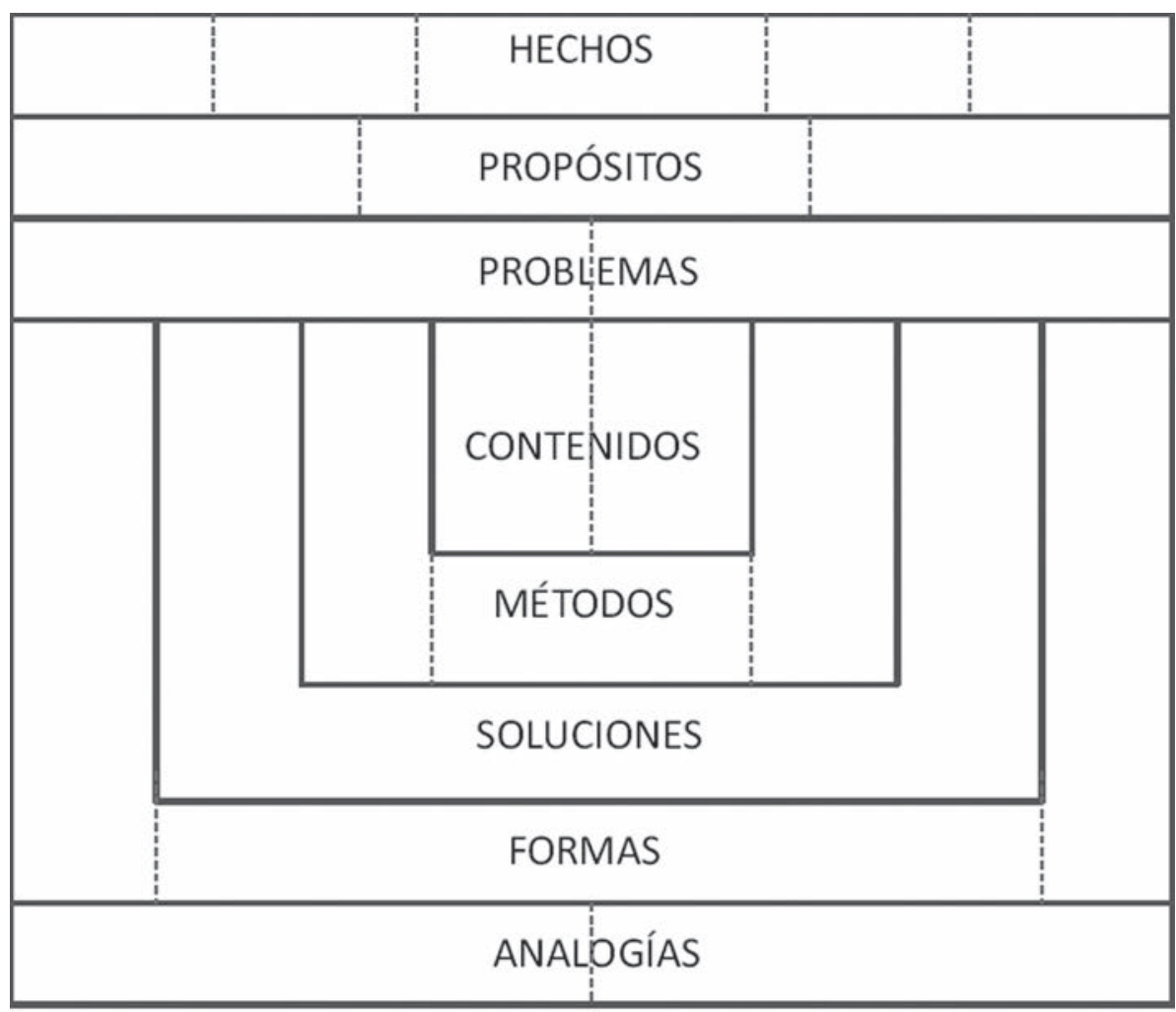


Este Prácticum Reflexivo puede ser aplicado en cualquier tipo de asignatura. De hecho algunos docentes del Departamento de Humanidades lo hemos puesto en práctica en nuestras clases de Filosofía Institucional y Epistemología.

\section{Referencias}

ALVARADO, Sara Victoria y otros (1992), Enfoques de la investigación en ciencias sociales, Investigación: Módulo 1, $2^{\mathrm{a}}$ ed. Medellín: CINDE.

ALVARADO, Sara Victoria y otros (1992), Proceso de construcción teórica, Métodos y técnicas en la investigación social, Investigación: Módulo 2, $2^{\mathrm{a}}$ ed. Medellín: CINDE.

ARNAL, Justo, DEL RINCÓN, Delio y LATORRE, Antonio (1992), Investigación educativa: Fundamentos y metodología. Barcelona: Labor.

BÁRCENA, F. y MÈLICH, J.C. (2000), La educación como acontecimiento ético. Barcelona: Paidós.

BERGER, P. L. y LUCKMANN, T. (1993), La construcción social de la realidad, $11^{a}$ reimpresión. Buenos Aires: Amorrortu Editores.

BLUMER, H. (1969), Symbolic interactionism. Perspective and Method, 1st pap. print. Berkeley, University of California Press, 1986.

BROCKBANK, A. y McGILL, I. (2002), Aprendizaje reflexivo en la educación superior. Madrid: Morata.

DEWEY, J.(1997), Democracia y educación. Madrid: Morata.

FACULTAD DE DERECHO, (2006), Reglamento general del Consultorio Jurídico. Bucaramanga, Universidad Santo Tomás.

FERNÁNDEZ CHRISTLIEB, P. (1994), La psicología colectiva un fin de siglo más tarde. Su disciplina. Su conocimiento. Su realidad. Santafé de Bogotá: Anthropos.

FOUREZ, G.(1998), La construcción del conocimiento científico. Madrid: Narcea.

GIROUX, H. A. (1988), Los profesores como intelectuales. Hacia una pedagogía crítica del aprendizaje. Barcelona, Paidós.

GOETZ, J. P. y LECOMPTE, M. D. (1988), Etnografía y diseño cualitativo en investigación educativa. Madrid: Morata.

GUTIÉRREZ MORÁN O.P. G. (2003), Treinta años de la seccional Bucaramanga. Apuntes breves para su historia.Bucaramanga, Universidad Santo Tomás.

HERNÁNDEZ, J.A. y CASTAÑEDA, D. H. (2009), Curso de filosofía del derecho. México: Oxford University Press.

JUNCO VARGAS, J.R. (1993), La Conciliación. Aspectos Sustanciales y Procesales. Santafé de Bogotá: Ediciones Jurídica Radar.

KLUG, U. (1951), Lógica jurídica. Bogotá: Temis.

MARTÍNEZ M. MIGUEL, La investigación cualitativa etnográfica en educación. Bogotá: Círculo de Lectura Alternativa, s.f.

MEAD, G.H. (1927: 1934, póstumo),(1972), Espíritu, persona y sociedad. Desde el punto de vista del conductismo social. Buenos Aires: Paidós, en FERNÁNDEZ CHRISTLIEB, P. (1994), La psicología colectiva un fin de siglo más tarde. Su disciplina. Su conocimiento. Su realidad. Santafé de Bogotá: Anthropos.

MÈLICH, J.C.(1994), Del extraño al cómplice. La educación en la vida cotidiana. Barcelona: Anthropos.

MONSALVE, A. (1992), Teoría de la argumentación. Medellín: Editorial Universidad de Antioquia.
MORIN, E. (1999), La mente bien ordenada. Barcelona: Seix Barral.

MURCIA FÉNIX, J.H.(2005), Sinopsis del Derecho Procesal Civil. Bogotá: Ediciones Doctrina y Ley Ltda.

NOT, L. (1998), Las pedagogías del conocimiento. Santafé de Bogotá: Fondo de Cultura Económica

NOT, L. (1992), La enseñanza dialogante. Barcelona: Herder.

NOVAK, J. D. (1982), Teoría y práctica de la educación. Madrid: Alianza Editorial.

PERELMAN, CH. (1976), Logique juridique. Nouvelle rhétorique. Paris: Dalloz.

PERELMAN, C. (1976), Logique juridique. Nouvelle rhétorique. Dalloz.

RICKERT BLUMER, H. (1969), Symbolic interactionism. Perspective

ROCHA OCHOA, C. (2006), Manual de introducción al Derecho. Bogotá: Editorial Unviersidad del Rosario.

SACRISTÁN, J. G. y PÉREZ GÓMEZ, A. I. (1994), Comprender y transformar la enseñanza. Madrid. Morata.

SCHÖN, D. (2002), La formación de profesionales reflexivos. Hacia un nuevo diseño de la enseñanza y el aprendizaje en las profesiones. Barcelona: Paidós.

SCHÖN, D. (1998), El profesional reflexivo. Cómo piensan los profesionales cuando actúan. Barcelona: Paidós.

SCHÖN, D. (2002), La formación de profesionales reflexivos. Hacia un nuevo diseño de la enseñanza y el aprendizaje en las profesiones. Barcelona: Paidós.

SCHÜTZ, A. (2000), La construcción significativa del mundo social. Introducción a la sociología comprensiva. Barcelona: Paidós

SOLANO SIERRA, J.E.(1994), Los términos procesales, $2^{\mathrm{a}}$. Ed.. Bogotá: Ediciones Doctrina y Ley.

STONE WISKE, M. (1999). La enseñanza para la comprensión. Vinculación entre la investigación y la práctica. Buenos Aires. Editorial Paidós.

TARAZONA, A. Estudiantes de Derecho. Historia del Consultorio Jurídico de la Universidad Santo Tomás de Bucaramanga. Monografía.

UNIVERSIDAD SANTO TOMÁS (2006), Reglamento General del Consultorio Jurídico. Bucaramanga: Departamento de Comunicaciones Universidad Santo Tomás.

CIBERGRAFÍA.

ATHERTON, J .S (2009) Learning and Teaching; SOLO taxonomy [On-line] UK: Available: http:// www.learningandteaching.info/learning/solo.htm Accessed: 21 February 2010

GALÁN MAÑAS, A. (2009), La enseñanza de la traducción en la modalidad semipresencial, Tesis doctoral. Barcelona, Universitat Autónoma de Barcelona, Departament de Traducció i d'Interpretació, en (062010): http://www.tesisenxarxa.net/TESIS UAB/ AVAILABLE/TDX-0416110-163024//aha1de2.pdf

GONZÁLEZ-SERNA, J.M.(2005), Las variedades temáticas del texto, en (06-2010): http://www.auladeletras. net/material/legales.PDF

THOMAS ARIAS, A. E. (2010). Nuevo régimen de prescripción civil, consultado en oct. 2010 , en http://www.javeriana.edu.co/Facultades/C Juridicas/pub_rev/documents/211-230.pdf

TORRE PUENTE, J.C. y GIL CORIA, E. (Eds) (2004), Hacia una enseñanza universitaria centrada en el aprendizaje Madrid, Universidad Pontificia Comillas, ps. 130-131, consultado $(2010,02)$ en: http://books google.com/books?id=HTgYrMpDBJIC\&pg = PA $130 \& \mathrm{lpg}=\mathrm{PA} 130 \& \mathrm{dq}=$ taxonomia + solo\&source $=$ bl\&ots=CIDsJjYagT\&sig=q7erdbR-LO VIrWK-Z9gB qy5 dz8\&hl $=$ encei $=0 k F 4 S 6 n r N 4 W W t g \bar{f} 40 S 7 \mathrm{Cg} \& s a$ $=\mathrm{X \& o} i=$ book result\&ct $=$ result\&resnum $=6 \& \mathrm{ved}=0$

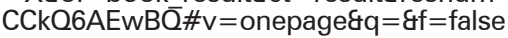

